\title{
Collaborative Landmark Mining With A Gamification Approach
}

\begin{tabular}{|c|c|}
\hline Florian Bockes & Matthias Ferstl \\
\hline University of Regensburg & University of Regensburg \\
\hline Universitätsstraße 31 & Universitätsstraße 31 \\
\hline 93053 Regensburg & 93053 Regensburg \\
\hline $\begin{array}{l}\text { Florian.bockes@stud.uni- } \\
\text { regensburg.de }\end{array}$ & $\begin{array}{l}\text { Matthias2.ferstl@stud.uni- } \\
\text { regensburg.de }\end{array}$ \\
\hline Laura Edel & Andreas Schmid \\
\hline University of Regensburg & University of Regensburg \\
\hline Universitätsstraße 31 & Universitätsstraße 31 \\
\hline 93053 Regensburg & 93053 Regensburg \\
\hline $\begin{array}{l}\text { Laura.Edel@stud.uni- } \\
\text { regensburg.de }\end{array}$ & $\begin{array}{l}\text { Andreas1.schmid@stud.uni- } \\
\text { regensburg.de }\end{array}$ \\
\hline
\end{tabular}

Permission to make digital or hard copies of part or all of this work for personal or classroom use is granted without fee provided that copies are not made or distributed for profit or commercial advantage and that copies bear this notice and the full citation on the first page. Copyrights for third-party components of this work must be honored. For all other uses, contact the owner/author.

MUM '15, November 30 - December 02, 2015, Linz, Austria

(c) 2015 Copyright held by the owner/author(s).

ACM ISBN 978-1-4503-3605-5/15/11.

DOI: http://dx.doi.org/10.1145/2836041.2841209

\begin{abstract}
In the field of pedestrian navigation some systems use prominent identifying features, so called landmarks. Attributes of high-quality landmarks are recognizability, actuality, uniqueness and noticeability. One of the challenges of this kind of navigation systems is to collect and evaluate landmarks with consistent quality. The system we developed solves these struggles with a crowdsourcing approach. We combine this with gamification elements in order to reach many users and to assure long-term motivation. Our system shows images of existing landmarks to the player, which he is afterwards asked to assign to a map of the university. Depending on the distance of his guess to the real position the player earns points. The application strives to encourage users to upload and rate pictures of existing landmarks. A multiplayer mode which allows challenging other users keeps them involved. In contrast to other products, our system does not rely on localization via GPS. Another goal was to implement a self-running system with a minimal amount of dedicated administration needed. Therefore the users with the highest scores are rating the submitted content.
\end{abstract}

\section{CCS Concepts}

- Information systems Database utilities and tools;

- Information systems Data mining; • Theory of 


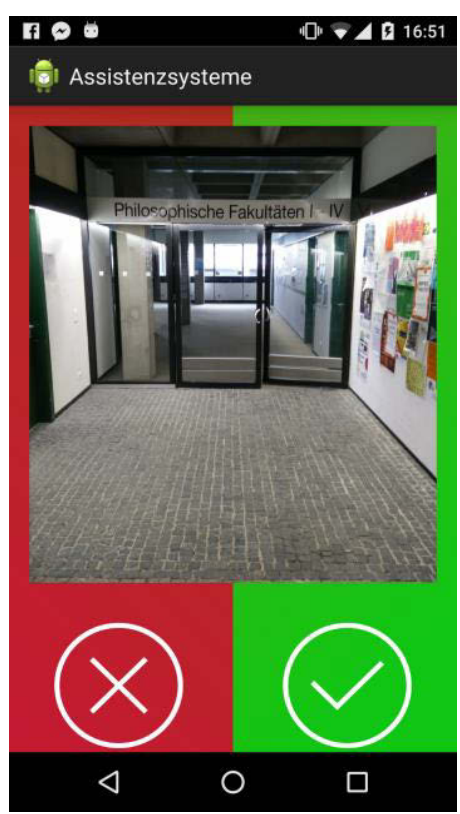

Figure 1: Game Screen 1 computation Convergence and learning in games;

\section{Author Keywords}

Landmarks; crowdsourcing; gamification

\section{Introduction}

Indoor navigation systems often suffer from the abstinence of recognizable landmarks. As for now, public buildings, like universities, often rely on plates and signposts to guide people to their destination. URWalking, a system developed by members of the University of Regensburg, tries to fix some of the most problematic aspects of indoor navigation by a different approach. It is based on the idea of "door-to-door" navigation. The goal is the situational support of pedestrians with a navigation system, which offers landmark-based route instructions [4]. Therefore URWalking needs landmarks of an overall high quality to ensure optimal navigation. A good landmark is defined by recognizability, actuality, uniqueness and noticeability [see 1]. In this paper we want to introduce a solution to collaborative landmark mining, in order to provide the best landmarks to the UR-Walking project.

\section{Concept}

According to Doan et al. [2] all projects which work with a crowdsourcing approach have to answer the same four questions:

1. How to acquire and retain participants?

2. What can people do?

3. How can the created content be combined?

4. How to deal with abuse?

Our answers consist of two software components. The first is the mobile application "URPicker" which shall be accessible for anyone interested. The second one is the corresponding administration tool which is accessible only for Supervisors. Question one, two and, in the final phase of the project, four should be answered by this game-like application. The import of the generated data to the UR-Walking system shall be solved by a cross system database which can be integrated. With the administration tool we can also control the content. Since the mobile application is a game, it should be easy to acquire players and keep them engaged. As research shows, games motivate people to work on challenging tasks with clear goals [6]. Those goals, collecting and evaluating landmarks, are communicated to the players, so they know their effort serves a higher purpose. The players will take photos of different landmarks and upload them with our application. After those pictures are rated by established user with a higher rank (supervisors), they will be shown to other participants. If they recognize the landmark, they have to locate them on a map. This part of the application serves the purpose of rating pictures of a landmark and the landmark itself indirectly. In contrast to web applications like geoguessr and earth picker [3,5] our application doesn't relay only on GPS data. In addition it isnt only for entertainment reasons, but also serves the purpose of landmark mining. Another important aspect of the project is the goal to reduce administrative workload for a single person to a minimum. Therefore there are two different types of players: Basic and Supervisor. Basic players have only access to the game. Players with a certain amount of points can achieve a supervisor status. They get access to the administration tool which comes along with new tasks like appreciation of submitted pictures or deleting obsolete landmarks. 


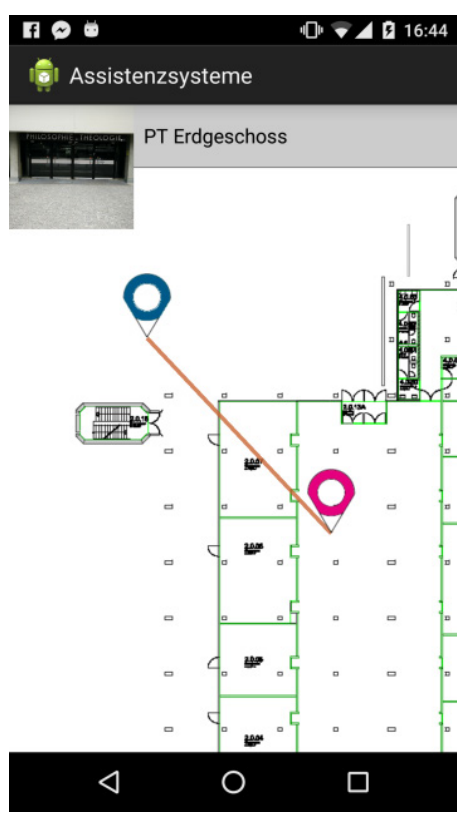

Figure 2: map visualisation

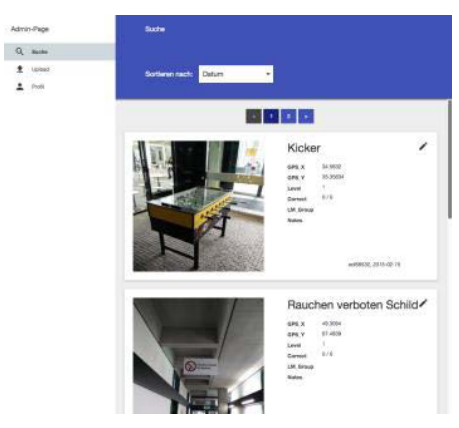

Figure 3: Admin Tool Search View
This all leads up to the goal to get a ranking of landmarks according to ratios of recognizability and accuracy, based on data we gather from all users. The best pictures taken by the users are shown in URWalking as navigation landmarks. Therefore we use the term gamification as explained by Deterding et. al [see 9] instead of serious games.

\section{Application}

The game was designed to be as simple as possible so that gameplay can start immediately. Figure 1 shows the first game screen. At the bottom of the screen points earned by the player are displayed and players can navigate to the main menu with the burger button. The player can decide by clicking on the corresponding button or with a swipe to the left or to the right whether he recognizes what is shown in the picture or not. The main menu offers the following interactions: Profile, Highscore, Record new landmark, Challenge, Options and Help. The functions which are displayed on the bottom during gameplay are now on the very top. Most of this functions are mandatory and need no further explanation but it should be noted that the design was tested in advance with a paper prototype and was well received by participants. In order to play the game there is no need for users to create an account. Registered users can individualize there profiles, store their highscore and can earn the supervisor status. The Application is targeted for Android 4.3+.

\section{Gameplay}

Our goal was to keep the user interface of the application as simple as possible. A Picture of a landmark or specific location on the campus is shown (Figure 1). The player is asked if he recognizes the shown landmark. If he does, the game goes on. Otherwise a new one is displayed and the process is started all over again until he marks a landmark as known. Afterwards the player is asked to mark the position on a campus map (Figure 2 ). It is possible to zoom into the map and even into buildings. According to the distance of the marked location and the accurate position, the player gets points. Besides recognizing landmarks and marking them accurately on the map every user can earn points by uploading new pictures and challenging other players. The ranking, which is based on the points, is the key part in motivating users. They are also used to determine if a player can participate in administration activities ("Supervisor" status).

The Challenge Mode is very similar to the normal game mode with a few exceptions. If player $A$ challenges player B and he/she accepts the challenge, both play a best of three game with the same landmarks. The winner gets the points of both players. This feature aims to increment the motivation of users interested in competitive challenges [see 7]. Since player motivation is a critical aspect of crowdsourced projects, we wanted to provide a better entertainment experience. Vorderer, Hartmann and Klimmt have shown this in their work [8].

\section{Admin Tool}

In addition to the android game we developed a tool to organize the landmark data (Figure 3-5). Supervisors are granted access to this tool in order to delete inappropriate content. This is done by voting for different landmarks. If a submission has three or more positive votes it is considered as appropriate landmark and will be released to the game mode. With those 


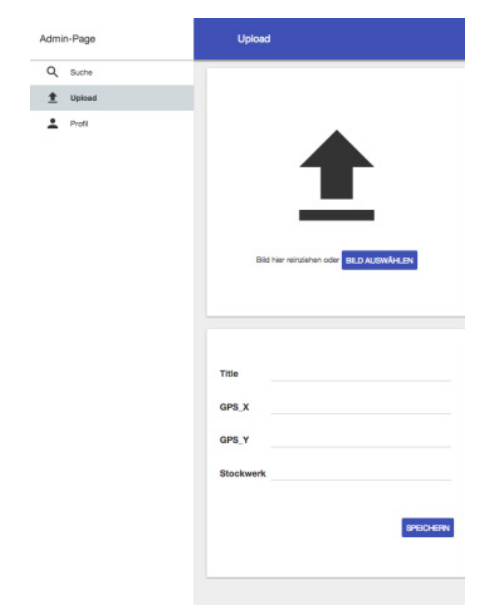

Figure 4: Admin Tool - Upload View 1

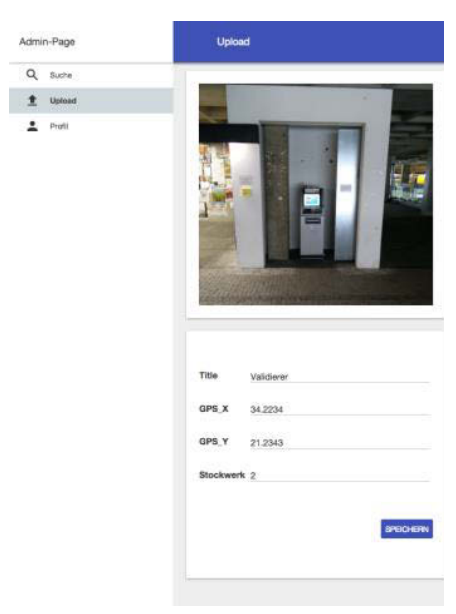

Figure 5: Admin Tool - Upload View 2 votes, supervisors also earn points to further encourage participation.

\section{Future Work}

First results of the system are still pending since the system has to be used by a critical amount of users over a certain amount of time. The current plan is to test the application with at least twenty users over a three Month timeframe. Afterwards we can evaluate the quality of certain landmarks according to the measurement of the metadata (e.g. percentage of recognition or accuracy of real location). The best landmarks can be integrated in UR-Walking. If this first test run is successful the concept has to be tested in other circumstances to prove its validity.

\section{Acknowledgements}

We want to thank Prof. Dr. Bernd Ludwig, Dipl.-Inf. Univ. Manuel Müller and M.A. Christina Ohm for their continuous support. Furthermore we want to thank other teams of the lesson "assistance systems" for their constructive feedback during the development of the system.

\section{References}

1. Andrew J. May, Tracy Ross, Steven H. Bayer, and Mikko J. Tarkiainen. 2003. Pedestrian navigation aids: information requirements and design implications. Personal Ubiquitous Comput. 7, 6 (December 2003), 331-338. http://dx.doi.org/10.1007/s00779-003-0248-5

2. Anhai Doan, Raghu Ramakrishnan, and Alon Y. Halevy. 2011. Crowdsourcing systems on the World-Wide Web. Commun. ACM 54, 4 (April 2011), 86-96.

http://doi.acm.org/10.1145/1924421.1924442
3. Anton Wallén. 2013. Geoguessr. Retrieved September 29, 2015 from https://geoguessr.com/

4. Bernd Ludwig, Manuel T. Müller, Christina Ohm. 2014. URwalking Retrieved September 29, 2015 from http://urwalking.ur.de/

5. Christian Altrichter. 2013. Earth picker. Retrieved September 21, 2015 from http://www. earthpicker.com/

6. Jane McGonigal. 2011. Reality is Broken: Why Games Make Us Better and how They can Change the World. Penguin Group, The.

7. Michael Sailer, Jan Hense, Heinz Mandl and Markus Klevers. 2013. Psychological Perspectives on Motivation through Gamification. Interaction Design and Architecture(s) Journal - IxD\&A, N.19, 2013, pp. 28-37.

8. Peter Vorderer, Tilo Hartmann, and Christoph Klimmt. 2003. Explaining the enjoyment of playing video games: the role of competition. In Proceedings of the second international conference on Entertainment computing (ICEC '03). Carnegie Mellon University, Pittsburgh, PA, USA, 1-9.

9. Sebastian Deterding, Dan Dixon, Rilla Khaled, and Lennart Nacke. 2011.From game design elements to gamefulness: defining "gamification". In Proceedings of the 15th International Academic MindTrek Conference: Envisioning Future Media Environments (MindTrek '11). ACM, New York, NY, USA, 9-15.

http://doi.acm.org/10.1145/2181037.2181040 